\title{
Quand le COVID-19 invite le numérique en psychomotricité \\ Pratiques et représentations du télésoin
}

\author{
Marie Agostinucci, Sylvain Hanneton et Bernard Andrieu
}

\section{Introduction}

Dès le 13 mars 2020, les mesures de confinement liées à l'épidémie de COVID-19 ont entrainé la suspension de nombreux soins en psychomoteurs, notamment dans le secteur libéral. Le $1^{\mathrm{er}}$ avril 2020, la Haute Autorité de Santé émis une fiche de recommandation des bonnes pratiques en télésoin et en téléconsultation.

Mais l'autorisation pour les psychomotriciens de le pratiquer n'y a été ajoutée que le 14 avril 2020 (Haute Autorité de Santé, 2020). Dans ce contexte de crise, cette discipline ancrée dans l'interaction des corps, doit s'adapter. La compatibilité entre le télésoin et l'exercice de la psychomotricité est ici questionnée à travers une enquête nationale auprès des psychomotriciens français.

\section{Une psychomotricité à distance}

\section{L'approche corporelle en psychomotricité}

2En France, l'exercice de la psychomotricité est réglementé par le décret de compétence n ${ }^{\circ} 88-659$ du 6 mai 1988 abrogé en 2004, portant sur l'accomplissement d'actes de rééducation des désordres psychomoteurs au moyen de médiations corporelles. Le psychomotricien s'intéresse donc à la structuration spatio-temporelle du mouvement, à la coordination, et à la maîtrise des gestes globaux et fins (Potel, 2010a). Historiquement marquée par des ancrages en neurologie et en psychiatrie (Potel, 2010b), cette discipline propose une approche de la motricité et du corps dans leur fonction relationnelle, au-delà de l'approche fonctionnelle (Rodriguez, 2014). Dans sa pratique, le psychomotricien s'appuie sur sa propre sensorialité dans l'échange avec le patient (Potel, 2010b). Dans le mouvement, le référentiel d'espace-temps devient le lieu d'expression du langage du corps (Potel, 2010b). La lecture phénoménologique du psychomotricien s'appuie sur les manifestations corporelles qu'il perçoit à partir de son propre corps (Rodriguez, 2014). En tant que métier du soin, la psychomotricité est aussi marquée par la prégnance du tact, cette qualité professionnelle définie comme une vertu à la base de la relation thérapeutique (Andrieu, 2007 ; Prairat, 2017). Par le toucher, les émotions sont engagées dans la rencontre (Andrieu, 2007), ainsi en psychomotricité il est déployé dans une dynamique tonico-émotionnelle. Le mouvement et le toucher sont donc les pierres angulaires de la pratique psychomotrice dont les médiations se déclinent autour du sport et des arts (Potel, 2010a). Pendant le confinement, certaines médiations comme la relaxation (Chevance et al., 2020) et l'activité physique (Ministère des Sports, 2020) sont d'ailleurs recommandées.

\section{Les enjeux du télésoin en situation de confinement}

3A l'échelle mondiale, le télésoin s'est présentée en solution d'urgence face au confinement (Li et al., 2020). Son intérêt majeur est d'offrir une forme plus ou moins satisfaisante de continuité des soins (Chevance et al., 2020 ; Pfefferbaum \& North, 2020). Les mesures sanitaires s'accompagnent de répercussions psychosociales et les études internationales alertent sur le risque de stress posttraumatique de ce type de configuration (Brooks et al., 2020 ; Liu et al., 2020). Les parents confinés avec leurs enfants sont d'ailleurs plus vulnérables et le risque chez les enfants en serait même multiplié 
$\begin{array}{llll}\text { par } & \text { (Buatre } & \text { et } & \text { 2020). }\end{array}$

L'anxiété, la dépression et les troubles du sommeil sont également rapportés (Brooks et al., 2020). Le télésoin est donc recommandé et déployé afin de fournir une psychoéducation et un soutien psychologique (Zhou et al., 2020). Bien que la pratique soit développée en Europe et à l'échelle internationale, la France n'autorise le télésoin en rééducation que depuis le mois d'avril 2020 en contexte de la pandémie à COVID-19 (Haute Autorité de Santé, 2020).

\section{Les rééducations à distance}

4La télémédecine se développe pour permettre un accès aux soins plus équitable et dans une logique de diminution des coûts, même si ce point reste peu documenté (Laver et al., 2020). Face aux déserts médicaux, elle constitue une solution de compensation sûre, efficace et confortable (Sarfo et al., 2018 ; Staszuk et al., 2016). Elle offre un accès aux soins aux personnes isolées ou présentant des difficultés de mobilité (Cox et al., 2018 ; Staszuk et al., 2016), et s'adapte aux patients peu disponibles du fait de leur activité professionnelle (Cox et al., 2018). Les modalités de télésoin sont variables: visioconférence, hotline téléphonique, application mobile, logiciel, environnement virtuel, monitoring (Cox et al., 2018; Russell, 2007; Zhou et al., 2020). La télé-rééducation se réfère à la dispensation de soins de rééducation via l'utilisation des technologies de la communication (Russell, 2007), pour l'entrainement et l'éducation thérapeutique (Bittner et al., 2015). Cette modalité de soin est encore récente, probablement en raison de la part importante du toucher et du guidage physique dans les thérapies manuelles (Russell, 2007). La littérature démontre qu'elle améliore l'état des patient et n'est pas moins efficace qu'un suivi ordinaire au regard de l'autonomie, de l'équilibre ou encore de la dépression (Laver et al., 2020 ; Piotrowicz et al., 2016 ; Sarfo et al., 2018). Elle est préconisée sous la supervision d'un thérapeute pour les rééducations longues ou impliquant un entrainement physique répétitif (Cox et al., 2018 ; Staszuk et al., 2016). La sévérité de la maladie est la condition majeure de limitation (Piotrowicz et al., 2016 ; Staszuk et al., 2016) et les contre-indications relèvent de : l'épilepsie, la spasticité, les troubles sensoriels ou les troubles de la conscience (Staszuk et al., 2016). Tous les patients ne maitrisent pas l'outils informatique et la présence physique peut être une condition de motivation (Piotrowicz et al., 2016), il est donc crucial de laisser le patient libre du choix de la modalité de soin (Piotrowicz et al., 2016) et d'envisager le télésoin comme une alternative thérapeutique (Cox et al., 2018).

\section{Méthode}

\section{Le design de l'enquête}

5Une enquête nationale sur la pratique de la téléconsultation en situation de pandémie à COVID-19 a été diffusée sur les réseaux sociaux et avec l'appui de la Fédération Française des Psychomotriciens par voie électronique. Le recueil des données a débuté le 3 avril 2020 et s'est clôturé le 11 mai 2020. L'enquête était initialement adressée à tous les rééducateurs, cependant les répondants sont quasiexclusivement des psychomotriciens. Pour cette raison les trois répondants qui appartiennent à d'autres professions ont été exclus de l'enquête.

\section{Le contenu}

6Les données socioéconomiques de base sont relevées au début du formulaire. Il comporte des questions fermées, des réponses à choix multiples et des questions ouvertes :

\begin{tabular}{|l|l|}
\hline Questions & Modalité \\
\hline $\begin{array}{l}\text { Maintenez-vous vos séances en présentiel pendant la période de } \\
\text { confinement? }\end{array}$ & Réponse fermée à choix unique \\
\hline
\end{tabular}




\begin{tabular}{|c|c|}
\hline Vos patients sont principalement : & Réponse fermée à choix multiples \\
\hline Les indications pour lesquelles ils vous consultent : & Réponse fermée à choix multiples \\
\hline Etes-vous à l'aise avec l'utilisation d'outil numérique en général? & Réponse fermée à choix unique \\
\hline $\begin{array}{l}\text { Etes-vous à l'aise avec l'utilisation d'outil numérique en contexte } \\
\text { de soin? }\end{array}$ & Réponse fermée à choix unique \\
\hline $\begin{array}{l}\text { Vous estimez-vous assez compétent et formé pour mettre en place } \\
\text { une procédure de téléconsultation? }\end{array}$ & Réponse fermée à choix unique \\
\hline $\begin{array}{l}\text { Aviez-vous déjà réalisé des séances en téléconsultation avant } \\
\text { l'épidémie de COVID-19? }\end{array}$ & Réponse fermée à choix unique \\
\hline Réalisez-vous des suivis en téléconsultation actuellement? & Réponse fermée à choix unique \\
\hline $\begin{array}{l}\text { Si oui, en moyenne combien de séances réalisez-vous par semaine } \\
\text { en téléconsultation : }\end{array}$ & Réponse fermée courte \\
\hline (Si oui) Selon quelles modalités? & Réponse fermée à choix multiples \\
\hline (Si oui) Sur quel type de durée ? & Réponse fermée à choix multiple \\
\hline Quels sont les outils que vous utilisez? & Réponse ouverte courte \\
\hline $\begin{array}{l}\text { Quels sont les outils que vous connaissez mais que vous n'utilisez } \\
\text { pas? }\end{array}$ & Réponse ouverte courte \\
\hline $\begin{array}{l}\text { Quelles sont les limites que vous rencontrez ou qui vous font penser } \\
\text { que la téléconsultation n'est pas possible dans votre cas : }\end{array}$ & $\begin{array}{l}\text { Réponse fermée à choix multiple } \\
\text { avec possibilité d'ajout d'une } \\
\text { réponse Autre }\end{array}$ \\
\hline Quels sont les bénéfices que vous trouvez à ce type de dispositif ? & $\begin{array}{l}\text { Réponse fermée à choix multiple } \\
\text { avec possibilité d'ajout d'une } \\
\text { réponse Autre }\end{array}$ \\
\hline $\begin{array}{l}\text { Etes-vous prêt à vous lancer dans l'expérience d'une séance en } \\
\text { visioconférence pendant la période confinement? (Avec vos } \\
\text { proches, votre famille ou vos amis) }\end{array}$ & Réponse fermée à choix multiple \\
\hline Avec qui ? (si non, passez) & Réponse fermée à choix multiple \\
\hline Pensez-vous que ce type d'initiative soit bienvenue? & Réponse fermée à choix multiple \\
\hline Souhaitez-vous ajouter quelque chose? & Réponse ouverte longue \\
\hline
\end{tabular}

\section{L'analyse statistique des données}

7Les effets traités sont mesurés à l'aide d'ANOVA et de tests post-hoc employant la correction de Bonferoni. Les réponses qualitatives présentée sous la forme d'un continuum ont été converties en données quantitatives afin d'effectuer des corrélations de Pearson : ainsi les réponses du type «Tout à 
fait » sont codées 4, et les réponses du registre «Pas du tout » sont codée 1. L'intervalle de confiance retenu pour l'ensemble des tests statistiques est de $95 \%$.

\section{Résultats}

\section{Les répondants et leur patientèle}

8Les participants sont principalement des femmes (159 contre 9 hommes), âgés en moyenne de 35 ans pour une répartition de 22 à 62 ans. Une majorité exerce en libéral (70 \%), mais les lieux d'exercices professionnels varient : secteur médico-social (30\%), milieu hospitalier ou clinique (22\%), secteur privé divers $(0,5 \%)$ et le secteur privé de la petite enfance $(0,3 \%)$. Certains psychomotriciens $(28,6 \%)$ cumulent deux secteurs d'activité : principalement une activité libérale associée à un exercice salarié dans le milieu médicosocial ou hospitalier. L'ensemble des régions française est représenté (à hauteur de 1 à 5 répondants par région) avec une prédominance de l'Ile de France (9\%). Les patients accompagnés par ces professionnels sont principalement des enfants et des adolescents (Graphique 1). Les principaux motifs de consultations sont les troubles des apprentissages scolaires, les troubles neurologiques, les troubles psychiatriques et l'accompagnement de la relation mère-enfant (Graphique 2).

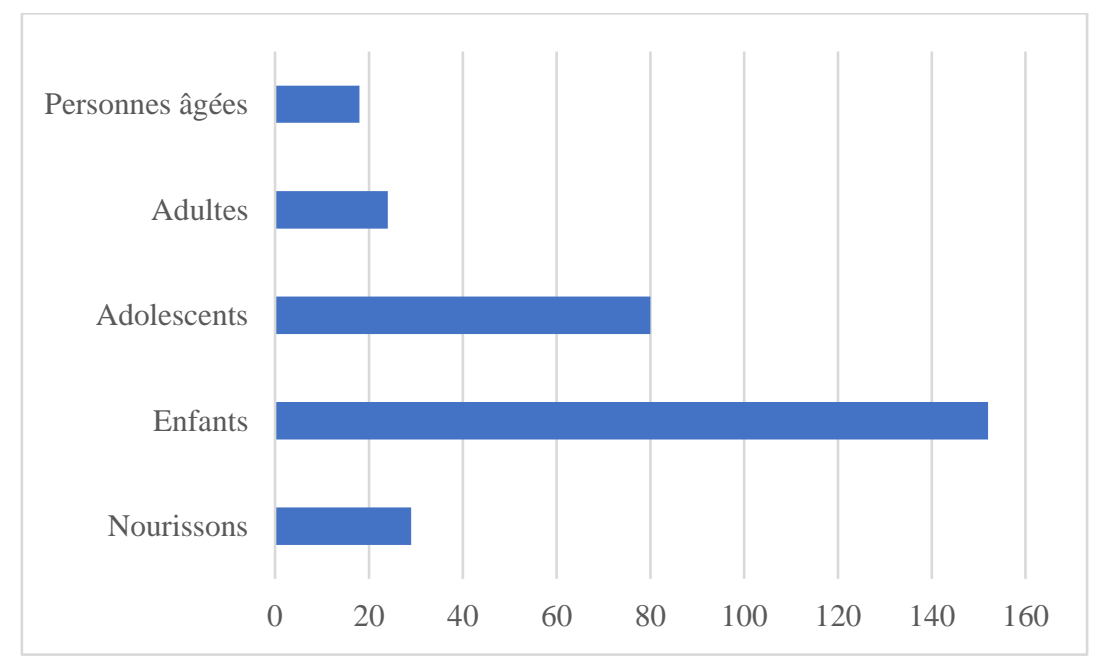

Graphique 1. Répartition de la patientèle

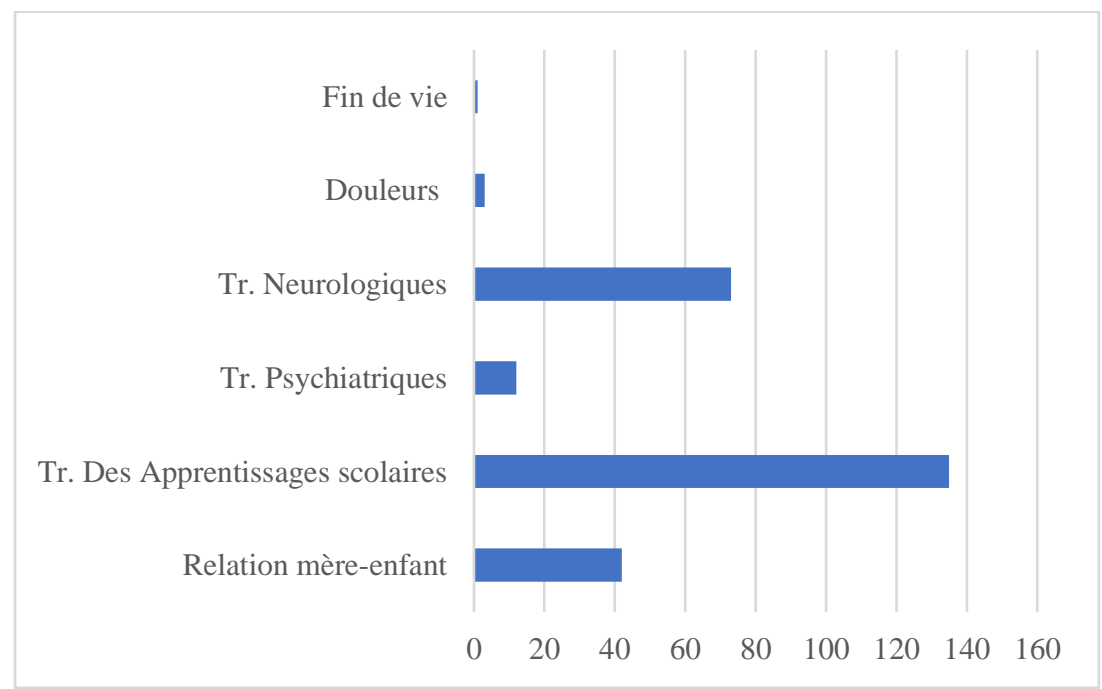

Graphique 2. Motifs de consultation 
En période de confinement, une majorité ne réalise plus de séance en présentiel $(86 \%)$. Seule une psychomotricienne a pu maintenir l'ensemble de ses suivis en contexte hospitalier, et 13,6\% des psychomotriciens ont maintenu les séances en présentiel de manière partielle.

\section{L'expérience des psychomotriciens en télésoin}

10La plupart des interrogés déclarent une bonne aisance dans le maniement des outils numériques en règle générale mais, d'après le résultat de la comparaison des variances et de l'ANOVA ( $\mathrm{p}$-value < 0,001 ), cette aisance diminue de manière significative lorsqu'il s'agit d'un contexte de soin (Graphique 3). Le test Post-Hoc (Tableau 1, Graphique 4), employé avec la correction de Bonferroni, révèle que cet effet est significativement différent pour des valeurs d'aisance en contexte général entre : être «Pas du tout à l'aise » et « Tout à fait à l'aise » (1-4), entre «Plutôt pas très à l'aise » et «Tout à fait à l'aise » (2-4), et entre « Plutôt à l'aise » et « Tout à fait à l'aise » (3-4). Le test de corrélation de Pearson confirme un lien significatif ( $\mathrm{p}$-value $<0,001, \mathrm{r}=0,32$ ) entre le degré d'aisance déclaré dans ces deux contextes.

Graphique 3. Aisance auto-estimée dans le maniement des outils numérique en contexte général et en contexte de soin

Post Hoc Comparisons - outilinfo

\begin{tabular}{ccccccc}
\hline & & Mean Difference & $\mathrm{SE}$ & $\mathrm{t}$ & $\mathrm{p}_{\text {tukey }}$ & $\mathrm{p}$ bonf \\
\hline 1 & 2 & -0.789 & 0.459 & -1.722 & 0.316 & 0.522 \\
& 3 & -1.042 & 0.433 & -2.408 & 0.080 & 0.103 \\
& 4 & -1.412 & 0.438 & -3.220 & 0.008 & 0.009 \\
2 & 3 & -0.253 & 0.185 & -1.362 & 0.525 & 1.000 \\
& 4 & -0.622 & 0.198 & -3.137 & 0.011 & 0.012 \\
3 & 4 & -0.370 & 0.128 & -2.885 & 0.023 & 0.027
\end{tabular}

Graphique 4. Degré d'aisance dans l'utilisation du numérique en contexte de soin en fonction de l'aisance en contexte général

Seulement 8 psychomotriciens avaient réalisé des téléconsultations avant le confinement. La majorité des répondants se sent d'ailleurs peu formée et peu compétente $(62 \%)$ pour mettre en place des téléconsultations (Graphique 4). L'ANOVA ne révèle pas d'effet significatif d'une expérience de télésoin antérieure sur le sentiment de compétence ( $\mathrm{p}$-value $>0,05)$, ni sur l'aisance déclarée en situation de télésoin ( $p$-value $>0,05$ ). L'application d'un test de Pearson révèle une corrélation forte entre le sentiment d'être formé ou compétent pour réaliser des téléconsultations et le degré d'aisance dans l'utilisation de l'outil numérique en contexte de soin ( $\mathrm{p}$-value $<0,001, \mathrm{r}=0,54$ ), et une corrélation faible entre le sentiment d'être formé ou compétent pour réaliser des téléconsultations et le degré d'aisance pour l'utilisation de l'outil numérique en général ( $\mathrm{p}$-value $<0,001, \mathrm{r}=0,26$ ). 


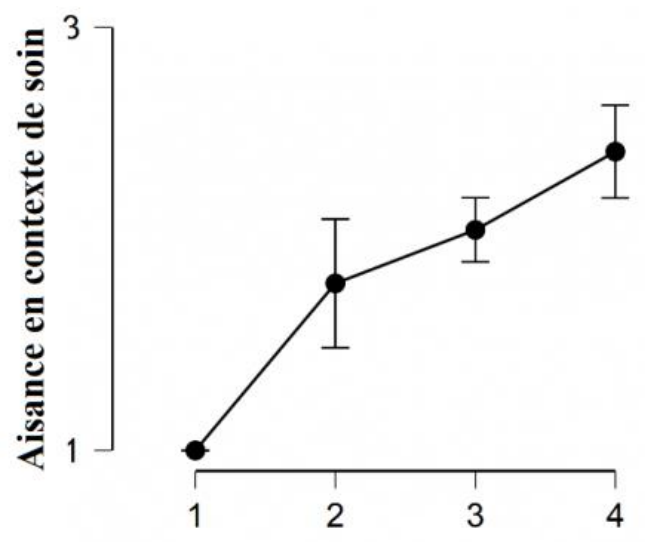

Aisance en contexte général

Graphique 4. Sentiment d'être formé ou d'être compétent pour réaliser des téléconsultations

\section{La pratique du télésoin en période de confinement}

Les psychomotriciens interrogés sont $42 \%$ à pratiquer des accompagnements en téléconsultation. Parmi ceux qui ne le pratiquent pas 34 professionnels déclarent que cette pratique est en projet, 8 n'ont pas reçu l'aval de leur direction, et 56 déclarent ne pas le pratiquer pour d'autres raisons (Graphique 5).

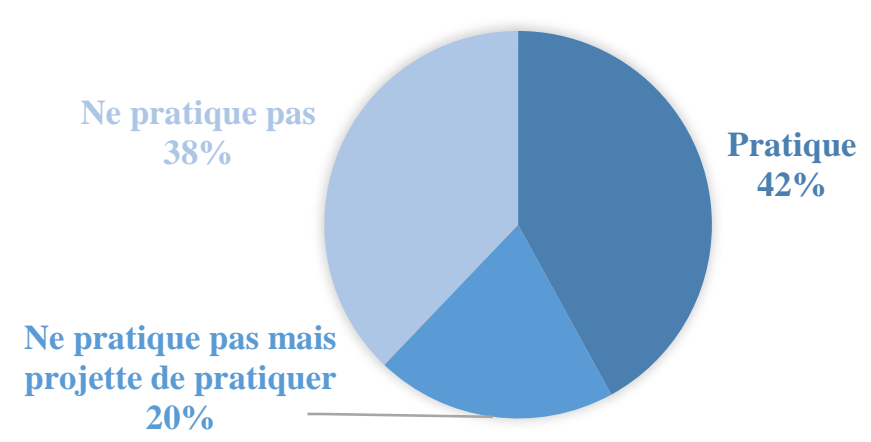

Graphique 5. Etat de la pratique de la téléconsultation en situation de confinement

13D'après les résultats de l'ANOVA, la pratique de la téléconsultation en situation de COVID-19, a un effet positif significatif sur le sentiment de compétence en télésoin ( $\mathrm{p}$-value $<0,001$ ) et sur l'aisance dans l'utilisation du numérique en télésoin ( $\mathrm{p}$-value $<0,005$ ). Le nombre de téléconsultations par semaine varie entre 1 et 27 mais la médiane est de 5. Elles sont réalisées dans le cadre d'accompagnement en individuel dans $96 \%$ des cas, bien que 8 professionnels réalisent aussi des accompagnements de groupe. La plupart des séances ont une durée comprise entre 30 minutes et une heure $(64,9 \%)$, dans d'autres cas elles durent entre 15 minutes et 30 minutes $(32,5 \%)$ et dans seulement deux cas elles sont inférieures à 15 minutes. L'ANOVA révèle un effet significatif de la durée des séances sur le sentiment de compétence ( $\mathrm{p}$-value < 0,005) ; d'après le test post-hoc réalisé avec la correction Bonferroni cet effet est significativement majoré au-delà de 30 minutes par séance (Tableau 2). Les plateformes utilisées sont très variables et il n'y a pas de logiciel qui soit significativement plus utilisé qu'un autre.

Post Hoc Comparisons - durée des séances 


$\begin{array}{llllll}\text { Mean } & \text { Lower } & \text { Upper } & \text { SE } & t & p_{\text {tukey }}\end{array}$

\begin{tabular}{|c|c|c|c|c|c|c|c|c|}
\hline \multirow[t]{2}{*}{$\begin{array}{l}\text { Entre } 15 \mathrm{~min} \text { et } \\
30 \mathrm{~min}\end{array}$} & $\begin{array}{l}\text { Entre } 30 \mathrm{~min} \text { et } \\
1 \text { heure }\end{array}$ & -0.480 & -0.867 & -0.093 & 0.162 & 2.967 & 0.011 & 0.012 \\
\hline & $\begin{array}{l}\text { Moins de } 15 \\
\text { min }\end{array}$ & 0.500 & -0.647 & 1.647 & 0.479 & 1.043 & 0.552 & 0.901 \\
\hline $\begin{array}{l}\text { Entre } 30 \text { min et } \\
1 \text { heure }\end{array}$ & $\begin{array}{l}\text { Moins de } 15 \\
\text { min }\end{array}$ & 0.980 & -0.144 & 2.104 & 0.470 & 2.086 & 0.100 & 0.121 \\
\hline
\end{tabular}

Confidence interval adjustment: tukey method for comparing a family of 3 estimates

Les principaux bénéfices identifiés sont : le maintien du lien thérapeutique (93\%), le soutien apporté pour mieux vivre le confinement (66\%) et le maintien du suivi (55\%). Les participants identifient en moyenne 6 limites. Les principales sont : le manque de contact relationnel (76\%), l'absence de contact physique (70\%), les contraintes matérielles du praticiens (30\%) et surtout du patient (67\%), la limite du cadre de la caméra (58\%), l'absence d'environnement spatial commun (49\%), la difficulté de maniement de l'outil numérique par le patient (39\%), la difficulté de visibilité des signes physiologiques (38\%), le manque de préparation et d'anticipation dans le déploiement du dispositif de télésoin (32\%) et la difficulté d'emploi de la musique en séance $(11,9 \%)$. Les autres difficultés mentionnées concernent l'absence du matériel habituel, la présence obligatoire d'un parent, et l'attention labile des enfants au domicile. Le nombre de limites identifié n'est pas influencé par la pratique d'après les résultats de l'ANOVA.Pour une pratique future de la téléconsultation en situation de pandémie à COVID-19, seuls $20 \%$ des répondants ne souhaitent pas mettre en place de téléconsultation. Ceux qui souhaitent débuter un suivi ou le poursuivre, déclarent qu'ils le feront avec leur patient (66\%) mais aussi auprès d'autres personnes comme leur proches $(35 \%)$.

\section{La question des valeurs}

La dernière question ouverte est dédiée à une expression libre. Dans ce cadre, trois psychomotriciennes ont présenté le télésoin comme un moyen de maintenir un lien thérapeutique et considèrent que cela «montre [son] soutien en tant que soignant ». Une autre estime qu'il a été autorisé trop tardivement et aurait dû être mis en place dès le mois de mars pour être vraiment pertinent. Une psychomotricienne met en relief le changement de perspective dans la relation thérapeutique :

"C'est intéressant car cela nous force à faire du lien avec les familles et à constater la réalité environnementale de nos patients. [...] au début, je me sentais parfois intrusive en appelant pour demander des nouvelles. J'ai finalement ouvert la possibilité de faire les séances en vidéos, et la plupart des patients (surtout les enfants) s'en sont saisis. Avec les ados je passe par le téléphone. J'ai été très positivement surprise par mes premiers essais : les enfants étaient attentifs, en lien, le cadre de la caméra permettait d'inventer de nouveaux jeux. Cela m'a encouragé à le proposer à plus de patients. »

Le soin a distance entre tout de même en conflit avec les valeurs de plusieurs psychomotriciens qui émettent des réserves voire de l'inquiétude sur ce nouveau mode d'exercice : « Mon métier ne s'y prête pas », «J'espère que l'usage de ces outils ne va pas se généraliser. Cela permet la continuité thérapeutique mais est extrêmement fatiguant quand on a choisi un travail avec l'humain loin des bureaux et ordinateurs », «Il ne faut pas s'engouffrer dans cette proposition de télésoin qui d'une part déshumanise, et ne permet pas de percevoir toutes les dimensions psychomotrices et émotionnelles 
mises en jeu. Le soin ce n'est pas être derrière un écran », «Dans cette période de confinement notre rôle n'est plus notre cœur de métier », « Le soutien des familles en visio, en appels et par SMS [...] je n'appelle pas ça un soin. Je ne facture pas ce soutien. Les parents ne peuvent être thérapeutes. On peut faire des exercices de rééducation supervisés à distance, c'est vrai. Mais mon métier n'est pas cela. », « le télésoin peut-il vraiment être considéré comme un soin psychomoteur dans la mesure où l'aspect relationnel mais surtout tout le tonico-émotionnel est omis sans l'échange avec notre outil principal... le corps ? », « téléconsultation difficile (à mon sens) dans un contexte de soin à partir du corps vécu, du travail de l'espace, de l'ajustement tonique etc. », « La part corporelle me semble difficile à aborder [...] Je suis inquiète d'une volonté de généralisation de ce mode d'exercice qui reste, même si intéressant dans certains cas, très limité ».Deux psychomotriciens pointent une incompatibilité entre le handicap de leurs patients et le télésoin dans le cas de l'autisme et du vieillissement. En revanche un psychomotricien l'utilisera pour accompagner les patients positifs au COVID.

\section{Discussion}

\section{Le devoir du soignant}

Le maintien du lien thérapeutique est largement mis en avant par les psychomotriciens, certains explicitent qu'il est important de fournir ce soutien à titre de soignant et dans une logique de solidarité. L'accompagnement au confinement est également très présent, d'autant que les patients concernés ici sont principalement des enfants et des adolescents confinés avec leurs parents. Ce soutien est d'ordinaire assumée physiquement par le psychomotricien, mais comme le décrit Eirick Prairat (2017) dans son article sur le tact : «Parler, c'est certes adresser un message, mais c'est aussi toucher ». Ces initiatives s'accordent avec les recommandations pour la prévention des risques psychosociaux auxquels les familles confinées sont particulièrement vulnérables (Brooks et al., 2020 ; Li et al., 2020). Cependant plusieurs psychomotriciens ne considèrent pas ce soutien comme un soin en psychomotricité.

\section{Un apprentissage dans l'urgence}

L'enquête permet de conclure que les individus qui maitrisent les outils numériques en général se sentent plus à l'aise dans l'utilisation de l'outil informatique en situation de soin que les autres, même s'ils présentent une aisance diminuée dans ce contexte. Les psychomotriciens qui pratiquent des téléconsultation de plus de 30 se sentent plus compétents dans la pratique du télésoin. Cela suggère que l'aisance s'améliore avec la pratique. Ainsi, si ce type de dispositif d'urgence doit être remis en place à l'avenir, il serait intéressant d'en maintenir l'autorisation en contexte ordinaire pour permettre aux soignants de développer leurs compétences.Certains psychomotriciens ont d'ailleurs eu pour objectif de proposer des séances à leurs proches dans le but de s'entrainer. Le développement de formation serait également bienvenu car le sentiment d'être compétent ou formé est orientée vers des lacunes, même si les psychomotriciens sont enclin à proposer du télésoin en contexte d'urgence. Comme le mentionne une participante, les liens avec les aidants sont modifiés mais l'adaptation peut devenir stimulante et intéressante. Cependant ce mode d'exercice nécessite des efforts de créativité et d'organisation, dans un contexte où les professionnels n'ont pas eu assez de temps pour se préparer et se former.

\section{Le conflit de valeurs}

Le télésoin est perçu comme peu approprié à la dynamique en psychomotricité. Dans une profession centrée sur la fonction relationnelle de la motricité et autant articulée autour du toucher, la rupture est profonde. Les gestes professionnels qui constituent la matrice identitaire et la légitimité du professionnel (Burel \& Andrieu, 2014) sont métamorphosés. Le toucher est un style de communication (Andrieu, 2007) à la base de son exercice, dont le psychomotriciens se voit privé. De plus, la lecture phénoménologique s'appuie sur les manifestations corporelles (Rodriguez, 2014); or la visibilité des signes physiologiques est limitée. Par ailleurs, la lecture du mouvement s'inscrit d'ordinaire dans un référentiel spatio-temporel commun au thérapeute et au patient (Potel, 2010b), or en situation de télésoin 
les protagonistes sont dans des espaces distincts. Ici, l'espace habité par l'enfant n'est plus celui habité par le thérapeute, Anne-Marie Gosselin (2002) décrit pourtant dans son article sur le jeu, l'importance cruciale de l'engagement du psychomotricien en terme de présence à l'enfant. Le soin à distance est donc peu adapté à l'exercice de la psychomotricité et ne s'accorde pas au sens qu'ils donnent à leur métier. Il résulte donc un conflit à d'aborder sous l'angle d'une clinique basée sur les valeurs (Plagnol, 2017). Certains participants manifestent d'ailleurs une crainte dans la généralisation du télésoin, même s'ils le tolèrent en contexte de crise.Bien que le niveau de preuve en télé-rééducation soit satisfaisant, les psychomotriciens posent la question des valeurs dans une approche personnalisée basée sur la singularité des individus (Plagnol, 2017). Comme le défend la littérature, le télésoin ne doit pas être envisagé en première intention mais comme une alternative pour des patients qui n'auraient pas accès aux soins et qui serait intéressés par cette modalité (Cox et al., 2018). Elle rejoint ainsi l'avis de ceux qui invitent explicitement à la modération dans le déploiement du télésoin. D'autres pointent tout comme la littérature, son incompatibilité avec certains handicaps (Piotrowicz et al., 2016 ; Staszuk et al., 2016).

\section{Conclusion}

La situation de confinement a conduit de nombreux psychomotriciens à développer une pratique de télésoin, principalement tournée vers le soutien psychologique aux familles et la préservation du lien thérapeutique. Cette pratique est marquée par un manque d'anticipation, partiellement compensé par un apprentissage sur le tas des psychomotriciens. Pour un déploiement optimal à l'avenir, il serait pertinent d'autoriser cette pratique en situation ordinaire et de développer des formations. L'exercice de la psychomotricité à distance se heurte aussi à un conflit de valeur puisque la dimension virtuelle altère la dynamique relationnelle inter-corporelle. Enfin, la littérature rejoint la réserve des psychomotriciens : le télésoin doit être déployé avec modération, comme une alternative thérapeutique pour les patients en rupture de soin.

Andrieu, B. (2007). Le soin de toucher. Le Portique. Revue de philosophie et de sciences humaines. http://journals.openedition.org/leportique/867

Bittner, A. K., Wykstra, S. L., Yoshinaga, P. D., \& Li, T. (2015). Telerehabilitation for people with low vision. Cochrane Database of Systematic Reviews, 8.

DOI : $\underline{10.1002 / 14651858 . C D 011019 . p u b 3}$

Brooks, S. K., Webster, R. K., Smith, L. E., Woodland, L., Wessely, S., Greenberg, N., \& Rubin, G. J. (2020). The psychological impact of quarantine and how to reduce it : Rapid review of the evidence. The Lancet, 395(10227), 912-920. https://doi.org/10.1016/S01406736(20)30460-8

DOI : $\underline{10.1016 / S 0140-6736(20) 30460-8}$

Burel, N., \& Andrieu, B. (2014). IntroductionVers une analyse du corps entier. Recherches \& éducations, 12, 9-12.

Chevance, A., Gourion, D., Hoertel, N., Llorca, P.-M., Thomas, P., Bocher, R., Moro, M.-R., Laprévote, V., Benyamina, A., Fossati, P., Masson, M., Leaune, E., Leboyer, M., \& Gaillard, R. (2020). Assurer les soins aux patients souffrant de troubles psychiques en France pendant l'épidémie à SARS-CoV-2. L'Encéphale.

Cox, N. S., McDonald, C. F., Hill, C. J., O’Halloran, P., Alison, J. A., Zanaboni, P., Macdonald, H., \& Holland, A. E. (2018). Telerehabilitation for chronic respiratory disease. Cochrane Database of Systematic Reviews, 6.

DOI : $\underline{10.1002 / 14651858 . C D 013040}$ 
Gosselin, A.-M. (2002). Le jeu du rééducateur. Recherches \& éducations, 3, Article 3. http://journals.openedition.org/rechercheseducations/307

Haute Autorité de Santé. (s. d.). Réponses rapides dans le cadre du COVID-19 Téléconsultation et télésoin. Haute Autorité de Santé. Consulté 14 mai 2020, à l'adresse https://www.has-sante.fr/jcms/p_3168867/fr/reponses-rapides-dans-le-cadre-du-covid-19teleconsultation-et-telesoin

Laver, K. E., Adey-Wakeling, Z., Crotty, M., Lannin, N. A., George, S., \& Sherrington, C. (2020). Telerehabilitation services for stroke. Cochrane Database of Systematic

Li, W., Yang, Y., Liu, Z.-H., Zhao, Y.-J., Zhang, Q., Zhang, L., Cheung, T., \& Xiang, Y.-T. (2020). Progression of Mental Health Services during the COVID-19 Outbreak in China. International Journal of Biological Sciences, 16(10), 1732-1738.

DOI : $\underline{10.7150 / \mathrm{ijbs} .45120}$

Liu, N., Zhang, F., Wei, C., Jia, Y., Shang, Z., Sun, L., Wu, L., Sun, Z., Zhou, Y., Wang, Y., $\&$ Liu, W. (2020). Prevalence and predictors of PTSS during COVID-19 outbreak in China hardest-hit areas : Gender differences matter. Psychiatry Research, 287, 112921.

Ministère des Sports. (2020, avril 21). Coronavirus COVID-19 : Avec le ministère des Sports, faire du sport chez soi, c'est facile ! sports.gouv.fr.

Pfefferbaum, B., \& North, C. S. (2020). Mental Health and the Covid-19 Pandemic. New England Journal of Medicine, 0(0).

Piotrowicz, E., Piepoli, M. F., Jaarsma, T., Lambrinou, E., Coats, A. J. S., Schmid, J.-P., Corrà, U., Agostoni, P., Dickstein, K., Seferović, P. M., Adamopoulos, S., \& Ponikowski, P. P. (2016). Telerehabilitation in heart failure patients: The evidence and the pitfalls. International Journal of Cardiology, 220, 408-413.

Plagnol, A. (2017). Justification des pratiques et clinique fondée sur les valeurs. Motricité Cérébrale : Réadaptation, Neurologie du Développement, 38(1), 13-20.

Potel, C. (2010a). Cinq axes essentiels de l'intervention en psychomotricité. In Etre psychomotricien : Un métier du présent, un métier d'avenir (p. 309-320). Erès.

Potel, C. (2010b). Un métier en lien avec son histoire et son environnement. In Etre psychomotricien : Un métier du présent, un métier d'avenir (p. 55-68). Erès.

Prairat, E. (2017). Reconsidérer le tact. Recherches \& éducations, 17, Article 17. http://journals.openedition.org/rechercheseducations/3394

Rodriguez, M. (2014). Médiation corporelles en psychomotricité : Évolution des idées. In Où en est la psychomotricité ? Etat des lieux et perspectives (p. 37-42). IN PRESS.

Russell, T. G. (2007). Physical rehabilitation using telemedicine. Journal of telemedicine and telecare, 13(5), 217-220.

DOI : $\underline{10.1258 / 135763307781458886}$ 
Sarfo, F. S., Ulasavets, U., Opare-Sem, O. K., \& Ovbiagele, B. (2018). Tele-Rehabilitation after Stroke : An Updated Systematic Review of the Literature. Journal of Stroke and Cerebrovascular Diseases, 27(9), 2306-2318.

DOI : $10.1016 / j . j$ jstrokecerebrovasdis.2018.05.013

Staszuk, A., Wiatrak, B., Tadeusiewicz, R., Karuga-Kuźniewska, E., \& Rybak, Z. (2016).

Telerehabilitation approach for patients with hand impairment. Acta of Bioengineering and Biomechanics, vol. 18(nr 4).

Zhou, X., Snoswell, C. L., Harding, L. E., Bambling, M., Edirippulige, S., Bai, X., \& Smith, A. C. (2020). The Role of Telehealth in Reducing the Mental Health Burden from COVID-19. Telemedicine and e-Health, 26(4), 377-379.

DOI : $\underline{10.1089 / \text { tmj. } 2020.0068}$ 